\title{
Three dimensional echocardiography for the assessment of mitral valve disease
}

\author{
N Sutaria, D Northridge, N Masani, N Pandian
}

\section{Department of Cardiology, Western General Hospital, Edinburgh, UK \\ N Sutaria \\ D Northridge}

Department of Cardiology, University Hospital of Wales, Cardiff, UK N Masani

Department of Cardiology, New England Medical Centre, Boston, USA N Pandian

Correspondence to: Dr N Sutaria, Reseach Fellow in Cardiology, Western General Hospital, Crewe Road, Edinburgh EH4 2XU, UK N.Sutaria@ed.ac.uk

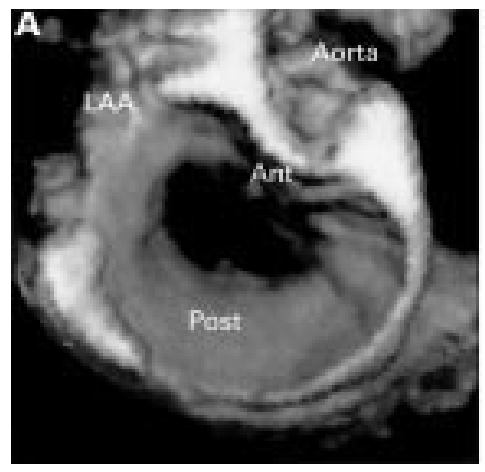

Figure $13 D E$ of the normal mitral valve. 3D images reconstructed from the left atrial (A) and left ventricular (B) perspectives, displaying fully the anterior (Ant) and posterior (Post) leaflets and their spacial relation with the aorta and left atrial appendage (LAA).

Three dimensional echocardiography (3DE) has been shown to be more accurate than $2 \mathrm{DE}$ in the quantification of cardiac volumes. ${ }^{12}$ These studies used either manually contoured, static "wire frame" reconstructions or dynamic "volumetric" automated reconstruction technology that is now commercially available-we concentrate on the latter methodology in this review. The benefits of 3DE are particularly its complex morphology and the importance of delineating its anatomy precisely in various pathological states. This was shown by Levine et al who used wire frame reconstruction of the mitral valve to define the $3 \mathrm{D}$ morphology of the mitral annulus and its relationship to mitral leaflet position, thereby clarifying the echocardiographic definition of mitral valve prolapse. ${ }^{3}$ The assessment of patients with mitral valve disease is one of the most promising clinical applications of this technology.

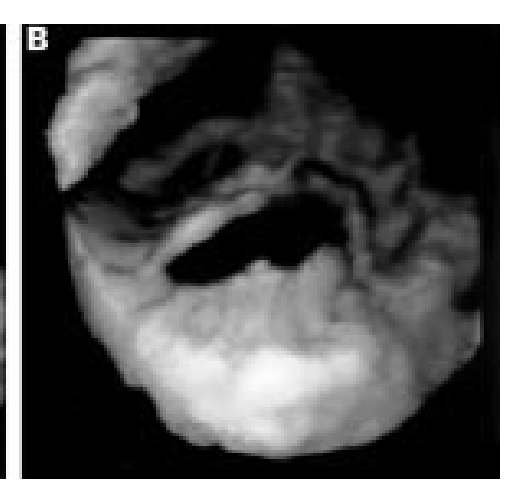

Whereas conventional two dimensional (2D) echocardiography is crucial to our understanding of the complex anatomy and three dimensional (3D) spacial relationships of cardiac structures, it requires the mental integration of a limited number of 2D imaging planes. This mental 3D reconstruction is inherently variable according to observer experience and expertise, and can only be described to other clinicians (such as surgeons) rather than displayed reproducibly. The display of cardiac anatomy in three dimensions from any perspective would have clear advantages over conventional $2 \mathrm{D}$ imaging and provide an insight into the functional and anatomic properties of cardiac structures. Recent advances in ultrasound and computer technology have been combined such that dynamic 3D echocardiographic imaging is now a practical reality. well suited to the study of the mitral valve given

.

\section{Dataset acquisition, processing and reconstruction}

A 3D dataset is composed of anatomical information from multiple $2 \mathrm{D}$ cross sectional images. For reconstruction of the mitral valve in adult patients, transoesophageal echocardiography (TOE) is the preferred approach for $2 \mathrm{D}$ image acquisition as it offers a relatively stable site for the imaging probe and superior resolution of the mitral valve apparatus. Images from a commercially available multiplane TOE probe are interfaced with a 3D computer system which incorporates the steering logic for acquisition of a rotational dataset and software for 3D reconstruction and display. With the mitral leaflets in the transverse midoesophageal view, the multiplane TOE probe is usually rotated at $2^{\circ}$ or $3^{\circ}$ increments over $180^{\circ}$ to give 90 or 60 sequential $2 \mathrm{D}$ cross sections, which are digitised to form a conical dataset. ${ }^{4}$ Optimal temporal and spacial registration is achieved by ECG and respiratory gating. Offline processing involves the conversion from polar to cubic Cartesian co-ordinates and interpolation of missing information between 2D slices. From the resultant dataset, novel 2D cut planes in any orientation can be selected (anyplane echo) and multiple parallel cross sectional 2D slices can be generated in any desired plane (paraplane echo). A volume rendered 3D image of the mitral valve can be reconstructed from any perspective (fig 1). Threshold limits are used to separate cardiac structures from blood pool and background. Brightness and shading provide perception of depth. With the added dimension of time we are able to study in detail the motion of the valve during the cardiac cycle.

\section{Mitral stenosis}

Three dimensional echocardiography has a role in both the quantitative and qualitative assessment of mitral stenosis. Three dimensional echocardiography overcomes the limitations of image plane positioning inherent in conventional $2 \mathrm{DE}$ and offers a more precise approach to measurement of mitral valve area. Paraplane 3DE allows the 2D short axis slice in the optimum plane of the orifice to be selected from the 3D dataset and the smallest complete orifice can be directly measured by planimetry (fig 2). Anterior leaflet calcification often produces acoustic shadows which obscure the mitral orifice in the $2 \mathrm{D}$ transthoracic short axis plane. Three dimensional TOE overcomes this problem by visualising the mitral valve from behind, so that acoustic shadows are cast into the left ventricle rather than over the leaflet tips. Chen et al assessed 15 patients with mitral stenosis and showed this 


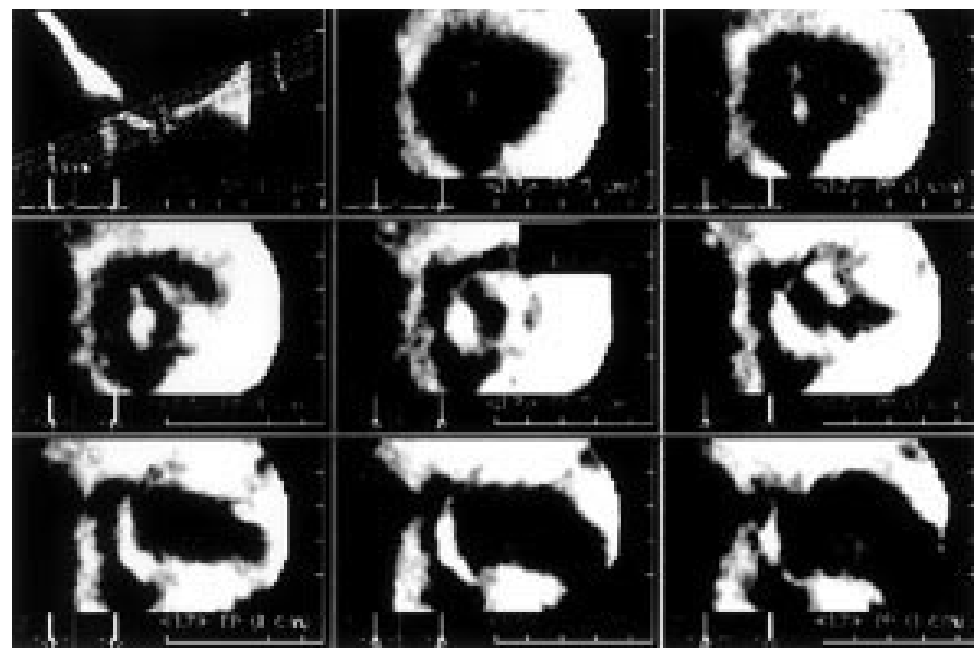

Figure 23 paraplane echo for measurement of mitral valve area. A series of parallel $2 D$ short axis cross sections are generated in the optimal plane of the mitral valve orifice. The $2 D$ slice defining the smallest complete orifice is selected and the valve area measured by planimetry.
$3 \mathrm{DE}$ also appears to be of value in the assessment of patients undergoing balloon mitral valvotomy (BMV) where the dominant mechanism is splitting of fused mitral commissures. Studies using $2 \mathrm{DE}$ have shown that commissural morphology is a powerful predictor of outcome after $\mathrm{BMV}^{9}$ and at our institution this forms the cornerstone of assessment of patients with mitral stenosis referred for valvotomy. Viewed from the left atrium, 3D reconstruction of mitral stenosis displays the restricted orifice, thickened leaflet margins and prominent left atrial appendage (fig 3A). In our experience, the volume rendered 3D display provides improved visualisation of mitral commissural fusion, particularly when the leaflets are viewed from the perspective of looking upwards from the left ventricle (fig 3B). Following balloon valvotomy, $3 \mathrm{DE}$ also defines clearly the extent and site of commissural splitting which may be symmetrical (fig 3C) or eccentric (fig 4). Other investigators have reported improved imaging of the mitral commissures with 3DE compared with $2 \mathrm{D}$ TOE.${ }^{10}$ Furthermore, $3 \mathrm{DE}$ assessment of commissural splitting following balloon inflation has been shown to relate to increase in mitral valve area. ${ }^{11}$ superior to $2 \mathrm{D}$ transthoracic echo for planimetry of mitral valve area when compared with the area measured at surgery with calibrated dilators. We have reported the first in vitro study to validate the accuracy of $3 \mathrm{D}$ TOE for the measurement of mitral valve area. ${ }^{7}$ In our study, porcine mitral valves were prepared with the commissures glued to simulate mitral stenosis. These valves were scanned in a water bath using $3 \mathrm{D}$ TOE. The reference standard valve area was determined using a digital photographic technique. Paraplane 3D TOE was shown to be more accurate than any previously described technique for quantifying mitral valve area and could become the "gold standard".

The shape of the mitral valve leaflets proximal to the orifice has an impact on the flow dynamics across a valve. 3DE with stereolithographic modelling has been used to demonstrate that flat shaped valves cause a higher pressure gradient for the same anatomic area and flow rate compared with "funnel" shaped valves. ${ }^{8}$ Thus, 3DE provides insights into mitral leaflet geometry which could refine our assessment of mitral stenosis.

\section{Mitral valve prolapse}

Mitral valve prolapse is a frequently encountered problem in clinical cardiology and the most common cause of isolated mitral regurgitation requiring surgical treatment. Mitral repair is associated with better outcome than valve replacement but demands a detailed understanding of valve morphology. The complex anatomy of the mitral valve makes interpretation of conventional 2D images difficult and is occasionally misleading. Dynamic volume rendered $3 \mathrm{DE}$ of the mitral valve displays both leaflets in their entirety from any desired perspective. This allows clear visualisation of the site and extent of leaflet prolapse during systole (figs 5 and 6). A number of studies have demonstrated the feasibility of $3 \mathrm{DE}$ in optimally displaying mitral valve prolapse. ${ }^{10}{ }^{12-14}$ The accuracy of $3 \mathrm{DE}$ in localising the involved leaflets has been confirmed at surgery. ${ }^{14}$ By elucidating the non-planar shape of the mitral annulus and its spacial relation to the mitral leaflets, 3DE might also improve the
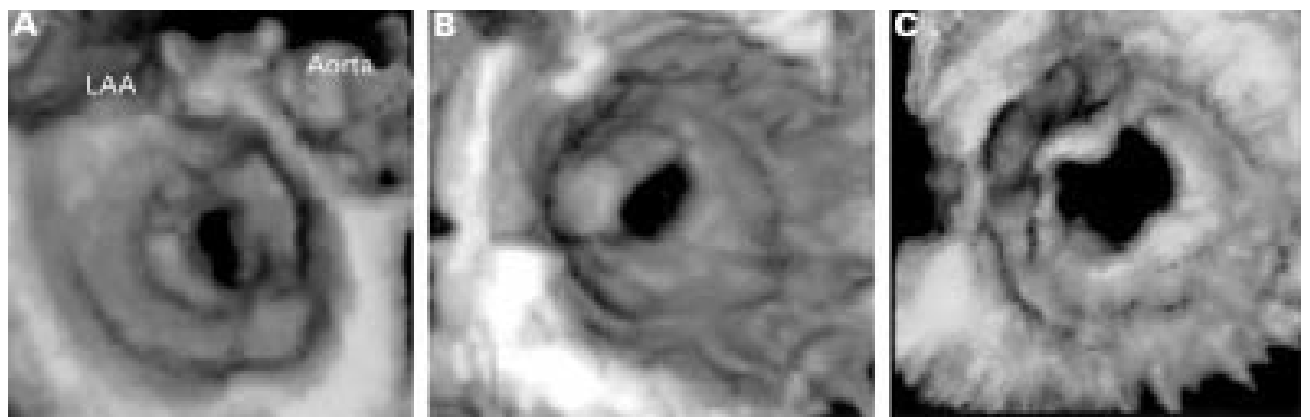

Figure $33 D E$ of rheumatic mitral stenosis. 3D images reconstructed from the left atrial $(A)$ and left ventricular (B) perspectives. The left ventricular view clearly shows thickened leaflets with a restricted orifice due to symmetrical commissural fusion. (C) shows the same valve after successful balloon mitral valvotomy demonstrating increase in orifice area due to bliateral commissural splitting. 
diagnostic sensitivity and specificity in mitral valve prolapse..$^{15}$ Reconstruction of the mitral valve en face from the left atrial perspective provides a view of the valve similar to that seen intraoperatively by the cardiac surgeon, with the major advantage of displaying the dynamic motion of the valve within the beating heart. ${ }^{12}$ Clearly, 3DE has great potential to facilitate the preoperative planning of mitral valve repair.

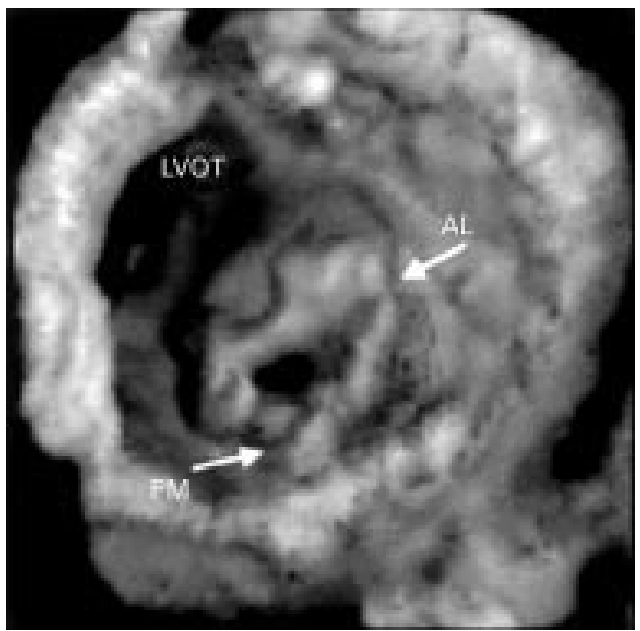

Figure $43 D E$ of mitral stenosis post balloon mitral valvotomy. $3 D$ images reconstructed from the left ventricular perspective. There has been splitting only of the posteromedial (PM) commissure, the anterolateral commissure (AL) remains fused resulting in an eccentric orifice. LVOT, left ventricular outflow tract.

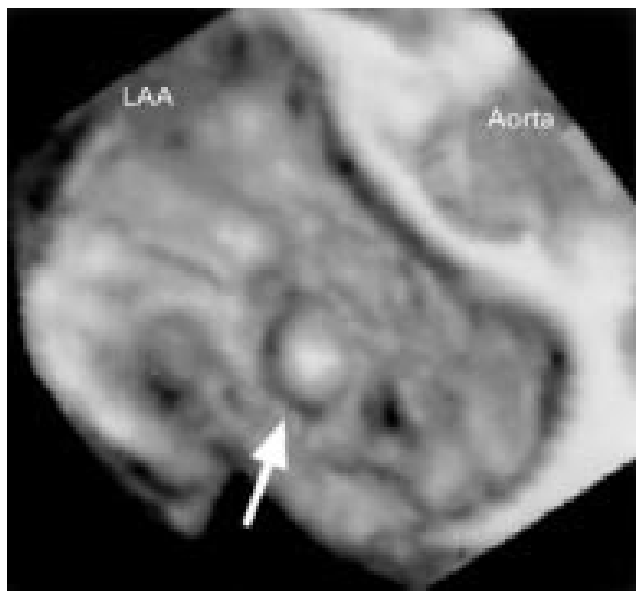

Figure 5 3DE of mitral valve prolapse: left atrial (surgical) view. Localised prolapse of the middle scallop of the posterior leaflet is seen bulging into the left atrium during systole (arrow).
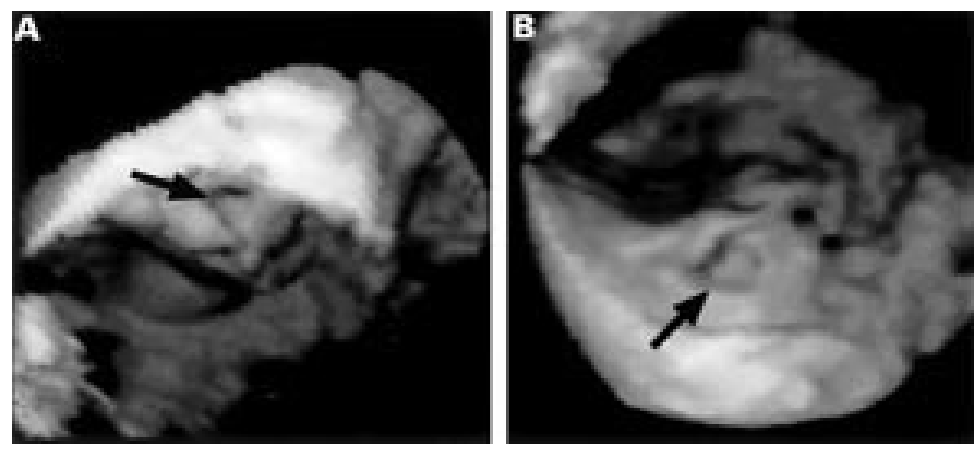

Figure 6 3DE of mitral valve prolapse: left ventricular view. Prolapse of the medial scallop of the posterior leaflet appears as an indentation (arrows).
With expected advances in computer technology and interactive software, we anticipate accurate simulation of surgery prior to repair operations in the form of "virtual repair".

\section{Mitral regurgitation}

As yet there is no widely accepted, non-invasive technique for accurate quantitation of mitral regurgitation. The regurgitant orifice in severe mitral regurgitation cannot be defined by $2 \mathrm{DE}$, and calculations based on Doppler flow are technically difficult and not widely applied. $3 \mathrm{DE}$ allows direct visualisation and planimetry of the regurgitant orifice in the optimal 2D plane, and measurements obtained by this method have been shown to correlate well with flow convergence derived measurements. ${ }^{16}$ However, this is only applicable in severe regurgitation. Recently, the ability to reconstruct Doppler colour flow has provided an insight into the mechanism and shape of mitral regurgitant jets. ${ }^{17-18}$ Three dimensional colour jet volume has been shown to correlate significantly with regurgitant volumes and may improve the assessment of eccentric MR because the full extent of these jets is better appreciated.

An important development in echocardiography has been the use of colour Doppler to evaluate the regurgitant flow convergence zone and calculate its proximal isovelocity surface area (PISA). Accurate measurement of the PISA would allow precise quantitation of mitral regurgitant volume, flow rate and regurgitant orifice. However, this method relies on assumptions regarding the geometry of the flow convergence zone-for example, that it is hemispheric. Cape et al have shown that the flow convergence zone is a complex $3 \mathrm{D}$ shape dependent on the shape of the regurgitant orifice ${ }^{19}$; this can be reconstructed precisely using $3 \mathrm{DE}$ in vitro $^{20}$ thereby measuring PISA directly, without geometric assumptions, upon which accurate calculations of flow rate can be based. The quantification of mitral regurgitant volume and effective regurgitant orifice area remain important yet elusive goals; we await clinical evidence to support these theoretical advantages of $3 \mathrm{DE}$.

\section{Limitations of three dimensional echocardiography}

The standard of the 3D reconstructed display depends critically on the quality of the original 2D cross sectional images. Until recently, in adult patients, this necessitated TOE. However, the development of harmonic imaging has made it feasible to reconstruct from a transthoracic rotational dataset. Minor movements of either patient or operator will distort the images and result in dropout which may be misinterpreted. Atrial fibrillation or a variable respiratory pattern prolongs the acquisition time and impairs the dataset resulting in artefact. Operator dependent changes in threshold settings, which define the tissueblood interface on the 3D rendered display, can affect the apparent mitral orifice. Therefore, measurements on reconstructed images should be made with caution. Spontaneous echo 
contrast in mitral stenosis hinders reconstruction of the valve from the left atrial perspective; this can be minimised by reducing the probe frequency. Highly mobile structures such as a ball valve thrombus, vegetations, and ruptured chords are not easily seen. In our opinion, $3 \mathrm{DE}$ has not improved visualisation of the mitral subvalvar apparatus and areas of calcification are not apparent in the volume rendered display. At present this technology provides information which complements that gained from a comprehensive 2D and Doppler echocardiographic study.

\section{Conclusions}

In the past, the major practical limitation of $3 \mathrm{DE}$ was the long time required for raw data processing and image reconstruction and suboptimal 2D image quality. With improved ultrasound technology, particularly harmonic imaging and faster digital processing, these problems are being overcome, and 3D software is being integrated into modern echo machines. A rotational 3D TOE dataset can be acquired, processed, and displayed within 10 minutes and has been shown to be feasible and useful in the intraoperative setting. "Real time" 3D transthoracic probes have been developed and are already commercially available. These factors will enhance the clinical applicability of $3 \mathrm{D}$ echocardiography in the future.

1 Sapin P, Schroeder K, Smith M, et al. Three dimensional echocardiographic measurement of left ventricular volume in vitro: comparison with two dimensional echocardiography and cineventriculography. $\mathcal{F} \mathrm{Am}$ Coll Cardiol 1993;22:1530-7.

2 Munoz R, Marcus E, Palacio G, et al. Reconstruction of 3-dimensional right ventricular shape and volume from 3 orthogonal planes. F Am Soc Echocardiogr 2000;13:177-85.

3 Levine R, Handschumaker M, Sandilippo A, et al. Levine R, Handschumaker M, Sandilippo A, et al.
Three-dimensional echocardiographic reconstruction of Three-dimensional echocardiographic reconstruction of
the mitral valve, with implications for the diagnosis of mitral valve prolapse. Circulation 1989;80:589-98.

4 Salustri A, Roelandt J. Three dimensional reconstruction of the heart with rotational acquisition: methods and clinical applications. Br Heart $\mathcal{F}$ 1995;73 (suppl 2):10-15.

5 Chen Q, Nosir Y, Vletter W, et al. Accurate assessment of mitral valve area in patients with mitral stenosis by three-dimensional echocardiography. $7 \mathrm{Am}$ Soc Echocardiography 1997;10:133-40.

6 Kaslival R, Trehan N, Mittal S, et al. A new 'gold standard' for the measurement of mitral valve area? Surgical validation of volume rendered three-dimensional echocardiography [abstract]. Circulation 1996;94(suppl):I-355.

7 Sutaria N, Shaw TRD, Fox KAA, et al. Three dimensional transoesophageal echocardiography for the measurement of mitral valve area: in vitro validation [abstract]. Heart 1999;81(suppl): P10,31.

8 Gilon D, Cape E, Handschumache M, et al. Insights from three-dimensional echocardiographic laser stereolithography. Effect of leaflet funnel geometry on the coefficient of orifice contraction, pressure loss and the Gorlin formula in mitral stenosis. Circulation 1996;94:452-9.

9 Fatkin D, Roy P, Morgan J, et al. Percutaneous balloon mitral valvotomy with the Inoue single-balloon catheter: commissural morphology as a determinant of outcome. 7 Am Coll Cardiol 1993;21:390-7.

10 Salustri A, Becker A, Herwerden L, et al. Three-dimensional echocardiography of normal and pathologic mitral valve: a comparison with two-dimensional transoesophageal echocardiography. $\mathcal{F}$ Am Coll Cardiol 1996;27:1502-10.

11 Applebaum R, Kasliwal R, Kanojia A, et al. Utility of threedimensional echocardiography during balloon mitral valvuloplasty. 7 Am Coll Cardiol 1998;32:1405-9.

12 Schwattz S, Cao Q, Azevedo J, et al. Simulation of intraoperative visualisation of cardiac structures and study of dynamic surgical anatomy with real time three-dimensional echocardiography. Am f Cardiol 1994;73:501-7.

13 Cheng T, Xie M, Wang X, et al. Evaluation of mitral valve prolapse by four-dimensional echocardiography. Am Heart f 1997;133:120-9.

14 Hozumi T, Yoshikawa J, Yoshida K, et al. Assessment of flail mitral leaflets by dynamic three-dimensional echocardiographic imaging. Am 7 Cardiol 1996;79:223-5.

15 Pai R, Tanimoto M, Jintapakorn W, et al. Volume rendered three-dimensional dynamic anatomy of the mitral annulus using a transoesophageal echocardiographic technique. $\mathcal{F}$ Heart Valve Disease 1995;4:623-7.

16 Breburda C, Griffin B, Pu M, et al. Three-dimensional echocardiographic planimetry of maximal regurgitant orifice area in myxomatous mitral regurgitation: intraoperative comparison with proximal flow convergence. $\mathcal{F}$ Am Coll Cardiol 1998;32:432-7.

17 Yao J, Masani N, Cao Q, et al. Clinical application of transthoracic volume-rendered three-dimensional echocardiography in the assessment of mitral regurgitation. Am $\mathcal{f}$ Cardiol 1998;82:189-96.

18 De Simone R, Glombitza G, Vahl CF, et al. Threedimensional color Doppler: a new approach for quantitative assessment of mitral regurgitant jets. $7 \mathrm{Am}$ Soc Echocardiogr 1999;12:173-85.

19 Cape E, Thomas J, Weyman A, et al. Three-dimensional surface geometry correction is required for calculating flow by the proximal isovelocity surface area technique. $f \mathrm{Am}$ Soc Echocardiogr 1995;8:585-94.

$20 \mathrm{Li} \mathrm{X}$, Shoita T, Delabays A, et al. Flow convergence flow rates from 3-dimensional reconstruction of color Doppler flow maps for computing transvalvular regurgitant flows without geometric assumptions: An in vitro quantitative flow study. F Am Soc Echocardiogr 1999;12:1035-44. 